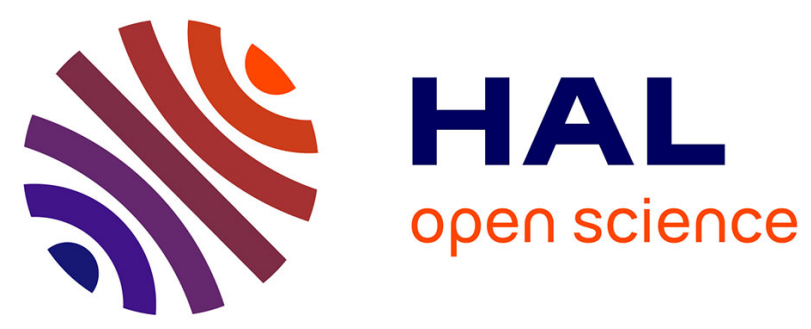

\title{
NUCLEAR REFRACTIVE EFFECTS IN NUCLEUS-NUCLEUS SCATTERING AT INTERMEDIATE ENERGIES
}

R. da Silveira, Ch. Leclercq-Willain

\section{- To cite this version:}

R. da Silveira, Ch. Leclercq-Willain. NUCLEAR REFRACTIVE EFFECTS IN NUCLEUSNUCLEUS SCATTERING AT INTERMEDIATE ENERGIES. Workshop on Semiclassical Methods in Nuclear Physics, 1984, Grenoble, France. pp.C6-395-C6-400, 10.1051/jphyscol:1984647 . jpa00224249

\section{HAL Id: jpa-00224249 https://hal.science/jpa-00224249}

Submitted on 1 Jan 1984

HAL is a multi-disciplinary open access archive for the deposit and dissemination of scientific research documents, whether they are published or not. The documents may come from teaching and research institutions in France or abroad, or from public or private research centers.
L'archive ouverte pluridisciplinaire HAL, est destinée au dépôt et à la diffusion de documents scientifiques de niveau recherche, publiés ou non, émanant des établissements d'enseignement et de recherche français ou étrangers, des laboratoires publics ou privés. 
R. da Silveira and Ch. Leclercq-Willain*

Division de Physique Théorique ${ }^{+}$, Institut de Physique Nucléaire, 91406 Orsay, France

* Physique Théorique, Université Libre de Bruxelles, 1050-Bruxelies, Belgium

Résumé - Nous donnons des arguments théoriques qui montrent la prësence d'un effet d'"arc-en-ciel" nucléaire dans la diffusion ēlastique $c^{12}+c^{12}$ à $1,016 \mathrm{GeV}$.

Abstract - We present theoretical evidence for the occurence of a nuclear rainbow effect in $\mathrm{C}^{12}+\mathrm{C}^{12}$ elastic scattering at $1.016 \mathrm{GeV}$.

I - INTRODUCTION. The separation of the "positive" and "negative" angle components from the experimental data.

The angular distribution of $\mathrm{C}^{12}+\mathrm{C}^{12}$ elastic scattering at $1.016 \mathrm{GeV} / 1,2 /$ has revealed a pattern similar to that already observed in $\alpha$-nucleus scattering at intermediate energies. In the latter case, the particular form of the angular distribution has been attributed $/ 3 /$ to a refractive effect due to a nuclear rainbow. The qualitative identification of a rainbow is based on the similarity between the angular distribution pattern and that of an Airy function. This procedure is qui te simple at low incident energies, because in this case only the coulomb rainbow is present $/ 4 /$. However, at intermediate energies, the nuclear transparency becomes large enough to reveal additional (nuclear) refractive contributions. As a result, the two contributing effects give rise to a complicated pattern due to inevitable wave-mechanical interferences and therefore the identification of the nuclear refractive contribution as a "rainbow", becomes ambiguous. In fact, in the resulting angular distribution only an exponential-like decrease, at large angles, is reminiscent of an Airy pattern. To avoid such difficulties, we shall proceed first to the elimination of the interference term from the angular distribution. This can be done by evoking the properties of the envelopes of the oscillatory pattern. These envelopes are defined by, $/ 5,6 /$

$$
E_{ \pm}(\theta)=\left[\sigma_{+}(\theta)^{1 / 2} \pm \sigma_{-}(\theta)^{1 / 2}\right]^{2}
$$

where $\sigma_{+}$and $\sigma$ are the scattering cross-section through "negative" and "positive" angles, "respectively. At each scattering angle $\theta$, the two values $E_{ \pm}$, allows us to obtain $\sigma_{ \pm}$from (1). The results we obtain $/ 6 /$, are shown in figures 1 and 2 , for elastic scattering of $\alpha$ on $\mathrm{Ca}^{40}(104 \mathrm{MeV}) / 7 /$ and $\mathrm{c}^{12}$ on $\mathrm{C}^{12}(1.016 \mathrm{GeV}) / 1 /$. The upper part of these figures, shows the experimental data as we 11 as the envelopes $E_{+}$. The two components $\sigma_{+}$are shown by open circles in 1 ower part of the figures. In both cases, the exponential-like component can be unambiguously identified as due to a coulomb rainbow, $i . e$. , the "positive" angle component. For $a+C a 40$, we immediatly recognize in the "negative" angle component, an Airy-type pattern, signature of a nuclear rainbow. However, for $C^{12}+C^{12}$, the interpretation of the $\sigma_{+} / \sigma_{R}$ component is considerably less clear.

\footnotetext{
+ Laboratoire Associé au C.N.R.S.
} 


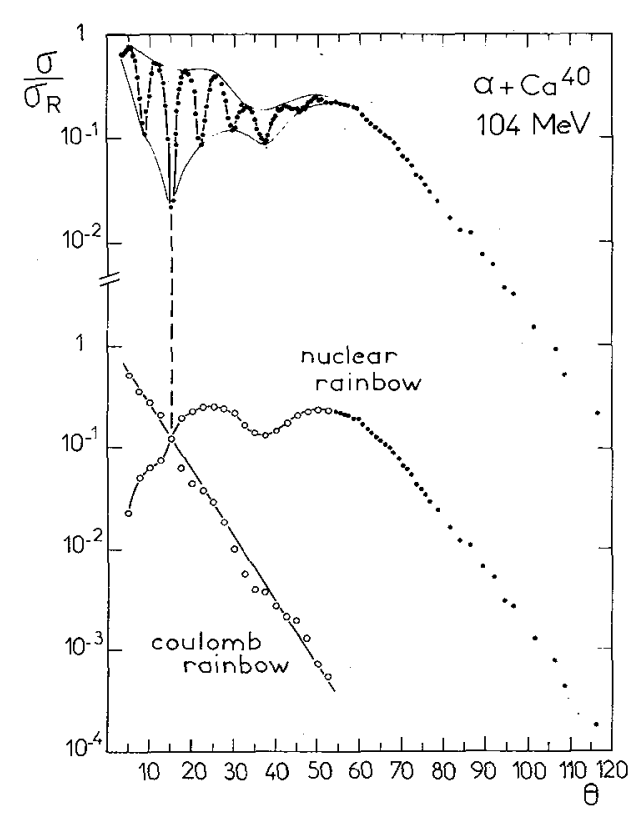

Fig.1

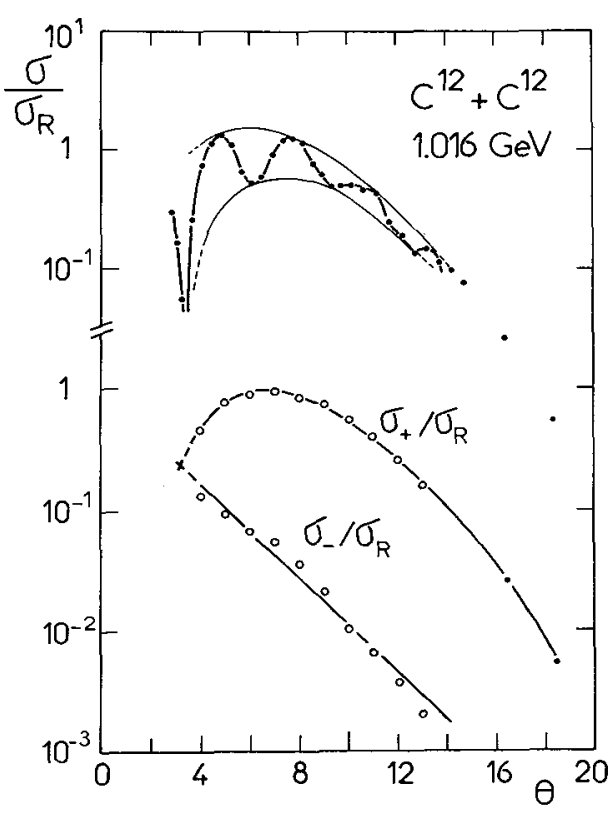

Fig.2

The aim of the present work is to clarify both qualitatively and quantitatively the origin of the "negative" angle component at intermediate energies $\mathrm{E} \widetilde{>} 100 \mathrm{MeV} / \mathrm{A}$. Particular attention should be paid to the relative importance of refractive and absorptive effects.

\section{II - The uniform asymptotic evaluation of the high energy scattering amplitude}

Assuming $V / E \ll 1$ ( $V$ is a measure of the absolute magnitude of the potential) and $k R \gg 1$, the scattering amplitude is written, in the $\ell$-representation $/ 8 /$,

$\left.f(\theta)=\frac{1}{i k} \int_{0}^{\infty} \mathrm{d} l \ell\right]_{0}(\ell \theta) \exp \left[-\frac{i}{\hbar v} \int_{-\infty}^{\infty} v_{o p}\left[\left(\ell^{2} / k^{2}+z^{2}\right)^{\frac{1}{2}}\right] d z\right]$

where $v_{0}=i W(r)+V(r)$ is the optical potential. The angular range of the approximation is ${ }^{\text {op }} \theta \sim V / E$. Furthermore, we assume that the regime of the scattering process is a quasi-classical one, i.e., /8/

$$
(V / E) \times K R \gg 1 \text { or } \theta k R \gg 1
$$

This condition allows the replacement of $J_{0}(x)$ by $(2 / \pi x)^{\frac{1}{2}} \cos (x-\pi / 4)$. The scattering amplitude becomes, $f(\theta) \sim f_{+}(\theta)+f_{-}(\theta)$. where $f_{ \pm}(\theta)$ are given by

$$
f_{ \pm}(\theta)=\frac{1}{i k}\left(\frac{1}{2 \pi \theta}\right)^{\frac{1}{2}} \int_{0}^{\infty} d \ell l^{\frac{1}{2}} e^{\omega(\ell)} e^{i \varphi_{ \pm}(l, \theta)}
$$

$$
\varphi_{ \pm}(\ell \cdot \theta)=\chi(\ell) \pm \ell \theta \mp \pi / 4
$$

$\omega(l)=\frac{1}{\hbar v} \int_{-\infty}^{\infty} W\left[\left(l^{2} / k^{2}+z^{2}\right)^{\frac{1}{2}}\right] d z, x(l)=-\frac{1}{\hbar v} \int_{-\infty}^{\infty} v\left[\left(l^{2} / k^{2}+z^{2}\right)^{\frac{1}{2}}\right] d z$ 
The square modutus of $f_{ \pm}(\theta)$ gives the scattering cross-section through "positive" and negative angles; ${ }^{ \pm} \sigma_{ \pm}(\theta)=\left|f_{ \pm}(\theta)\right|^{2}$. The exponential factor, exp $\omega(\ell)$, is the modulus of the $S(l)$ matrix, $|S(l)|=\exp \omega(l)$.

The $\sigma_{+}$component is obtained through the integral (4), in which $\omega(l)$ and $\chi(\ell)$ are supposed to be smooth varying functions of $\ell$. As is well known, in the classical limit, the integral (4) can be evaluated with high degree of accuracy by stationary phase methods. However, in the present case, the points of stationary phase can be either well separated or coalesce, when $\theta$ varies. To account for both situations, we use the uniform asymptotic approximation 19,10\%. The integral (4) is of the general form $/ 10 /$

$$
I(\alpha)=\int g(x) e^{i f(\alpha, x)} d x
$$

It is assumed that $f(\alpha, x)$ has two stationary points, $x=x^{(i)}(\alpha), i=1,2$, which can close together or coalesce when $\alpha$ varies. The uniform approximation of (7) is

$$
\begin{aligned}
& I \approx 2^{\frac{1}{2}} \pi e^{i A}\left[\left(G_{1}+G_{2}\right) \xi^{\frac{1}{4}} A i(-\xi)+i\left(G_{2}-G_{1}\right) \xi^{-\frac{1}{4}} A^{\prime} i(-\xi)\right] \\
& G_{1}=g\left(x^{(1)}\right) /\left[f^{\prime \prime}\left(\alpha, x^{(1)}\right)\right], \quad G_{2}=g\left(x^{(2)}\right) /\left[-f^{\prime \prime}\left(\alpha, x^{(2)}\right)\right] \\
& \xi=\left\{\frac{3}{4}\left[f\left(\alpha, x^{(1)}\right)-f\left(\alpha, x^{(2)}\right)\right]\right\}, A=\frac{1}{2}\left[f\left(\alpha, x^{(1)}\right)+f\left(\alpha, x^{(2)}\right)\right]
\end{aligned}
$$

where $A i$ and $A^{\prime} i$ are the Airy function and $i$ ts derivative, respectively. When the $x(i)$ are rea1, $\xi$ is positive, whereas when the $x(i)$ are complexe conjugate, $\xi$ is negative. The uniform approximation includes as special cases the non-uniform results : the stationary phase method $(\xi \gg 0)$ and the Airy approximation $(\xi \sim 0)$. Now, the stationary phase points of $\varphi_{f}(l, \theta)$, are those satisfying $\varphi_{f}^{\prime}(l, \theta)=0$, or

$-\frac{1}{\hbar v} \frac{d}{d \ell} \int_{-\infty}^{\infty} V\left[\left(\ell^{2} / k^{2}+z^{2}\right)^{\frac{1}{2}}\right] d z=\frac{1}{E} \int_{0}^{\infty} \frac{b}{r}\left[-v^{\prime}(r)\right] d z=-\theta, \quad b=\ell / k$

The left-hand side of (9) is the expression of the classical deflection angle /12/ in the limit $V / E \ll 1$. Equation (9) is written $\theta(l)=-\theta$. Therefore to evaluate $\sigma_{+}(\theta)$ we only need the negative part of the deflection function $\theta(\ell)$. We paramatrize $\theta(\ell)$ by

$$
\theta(\ell)=\theta_{r}+q\left(l-\ell_{r}\right)^{2}, \ell \sim \ell_{r}
$$

where $\theta_{r}, q$ and $\ell_{r}$ can be related to the parameters of the real part of the optical potential. The nuclear rainbow angle $\theta_{r}$, can be evaluated from (9). We take for $V(r)$ a Wood-Saxon shape, $V=-V_{0}[1+\exp (R-r) / a]^{-1}$. The force $f=-V^{\prime} r$ is almost negligible outside the range $R-a<r<R+a$, where it remains close to $-V^{\prime}(r) r=R=-V_{0} / 4 a$. Now, the maximum scattering angle, is reached by the trajectory of impact parameter $b=R-a$. Putting $b / r \sim 1$ in $(9)$, one abtains,

$$
\theta_{r} \sim-\frac{1}{2} \frac{V_{0}}{E}\left(\frac{R}{a}\right)^{\frac{1}{2}}
$$

The curvature of $\theta(\ell)$ in $\ell=\ell_{r}, q$, can be related to the thickness of the surface region $\Delta r=2 a$, through $/ 5 /$,

$$
q \sim \theta_{r} /(k \Delta r)^{2}=\theta_{r} / 4 a^{2} k^{2}
$$

Finally, in the limit $V / E \ll 1$ one has approximately $\ell_{r}=k R$. At this stage, we are able to appreciate at what extent the different conditions of the high energy approximation are fulfilled in the case of $\mathrm{C}^{12}+\mathrm{C}^{12}$ at $1.016 \mathrm{GeV}$. Taking for $V(r)$ the parameters obtained by Buenerd et a1./2/ from a phase shift analysis of the data, 
one obtains

$$
V_{0}=80 \mathrm{MeV}, R=3.66 \mathrm{fm}, \quad a=0.47 \mathrm{fm},
$$

$$
V_{0} / E \sim 0.16, \quad k R \sim 44, \quad V_{0} R / h v \approx 7, \theta_{r} \approx 0.175
$$

To proceed further, we now consider the particular form of $|S(\ell)|=\exp \omega(\ell)$. We shal1 use the familiar parametrization, $/ 11 /$,

$$
\exp \omega(\ell)=\left\{1+\exp \left[\left(\ell_{0}-\ell\right) / \Delta\right]\right\}^{-1}
$$

within the angular range not too far from $\theta_{r}$, one has $\ell \ll \ell_{0}$ and (13) can be approximated by

$$
\exp \omega(\ell) \sim \exp \left[\left(\ell-\ell_{0}\right) / \Delta\right]
$$

To remain consistent with the quasi-classical regime expressed by (3), the thickness $(\sim 2 \Delta)$ of (13) should be much larger than the uncertainty $\delta 2$ on $\ell$. The $\ell$-values in the range $\left|\ell-\ell_{0}\right| \sim 2 \Delta$ correspond to classical trajectories deviated through $\theta \sim \Delta p / p$. As $\delta l=k \delta b$ and $\delta b \Delta p \sim \hbar$, one has $\Delta \gg 1 / 2 \theta$, i.e., $\Delta \gg 1$.

Taking into account (10) and (14), one obtains from (8),

$$
\begin{aligned}
& \left|f_{+}(\theta)\right|^{2}=\sigma_{+}(\theta) \sim \frac{2 \pi \ell_{r}}{k^{2} \theta q^{\frac{2}{3}}} p^{2}\left(l_{r}-\ell_{0}\right) F_{\alpha}(x) \\
& F_{\alpha}(x)=A^{2} i(x)+S_{ \pm}^{2}(\alpha \sqrt{|x|})\left\{\frac{A^{\prime} i(x)}{|x|} \pm A^{2} i(x)\right\} \\
& x=\left(\theta-\left|\theta_{r}\right|\right) / q^{\frac{1}{3}}, \alpha=1 / \Delta q^{\frac{1}{3}} \quad p^{2}=e^{2\left(l_{r}-\ell_{0}\right) / \Delta}
\end{aligned}
$$

and,

$$
\begin{aligned}
& \text { (+) for } \theta<\left|\theta_{r}\right|, S_{+}=\sinh (\alpha \sqrt{|x|}) \\
& (-) \text { for } \theta>\left|\theta_{r}\right|, S_{-}=\sin (\alpha \sqrt{x})
\end{aligned}
$$

Further, we have taken for the classical cross-sections $\sigma_{1}$ and $\sigma_{2}$ associated with the two branches of $\theta(\ell)$, the mean value $\widetilde{\sigma}$

$$
\sigma_{1} \sim \sigma_{2} \sim \bar{\sigma}=\ell_{r} / \theta k^{2}\left|\theta^{\prime}\right|
$$

and for $\theta>\left|\theta_{r}\right|$, we have put

$$
|\ell|=\left[\ell_{r}^{2}+\left(\theta-\left|\theta_{r}\right|\right) / q\right]^{\frac{1}{2}} \sim \ell_{r}
$$

III - Analysis of the angular distributions. Application to $c^{12}+C^{12}$ scattering at $1.016 \mathrm{GeV}$.

To analyze the angular distributions, we shall take the product $\left[\theta x \sigma_{+}(\theta)\right]$ rather than $\sigma_{+}(\theta)$ itself. The advantage is, that the resulting pattern depends only on the reduced variable $x=\left(\theta-\left|\theta_{r}\right|\right) / q^{\frac{1}{3}}$. Consider the function $F_{\alpha}(x),(16)$. The graphical representation of $F_{\alpha}(x)$, is plotted in figure 3 , for different values of $\alpha ; \alpha=0 ., 0.5,1 ., 1.5,2 ., 2.5$. It is clear from figure 3 that the pattern evolution of $\dot{F}_{\alpha}(x)$ is determined by the parameter, $\alpha=1 / \Delta q^{\frac{1}{3}}$, which depends on both absorptive and refractive contributions, through $\Delta$ and $q^{\frac{1}{3}}$, respectively. For $\alpha \rightarrow 0, \Delta \rightarrow \infty$, one has,

$$
F_{\alpha}(x) \rightarrow A_{i}^{2}(x)
$$




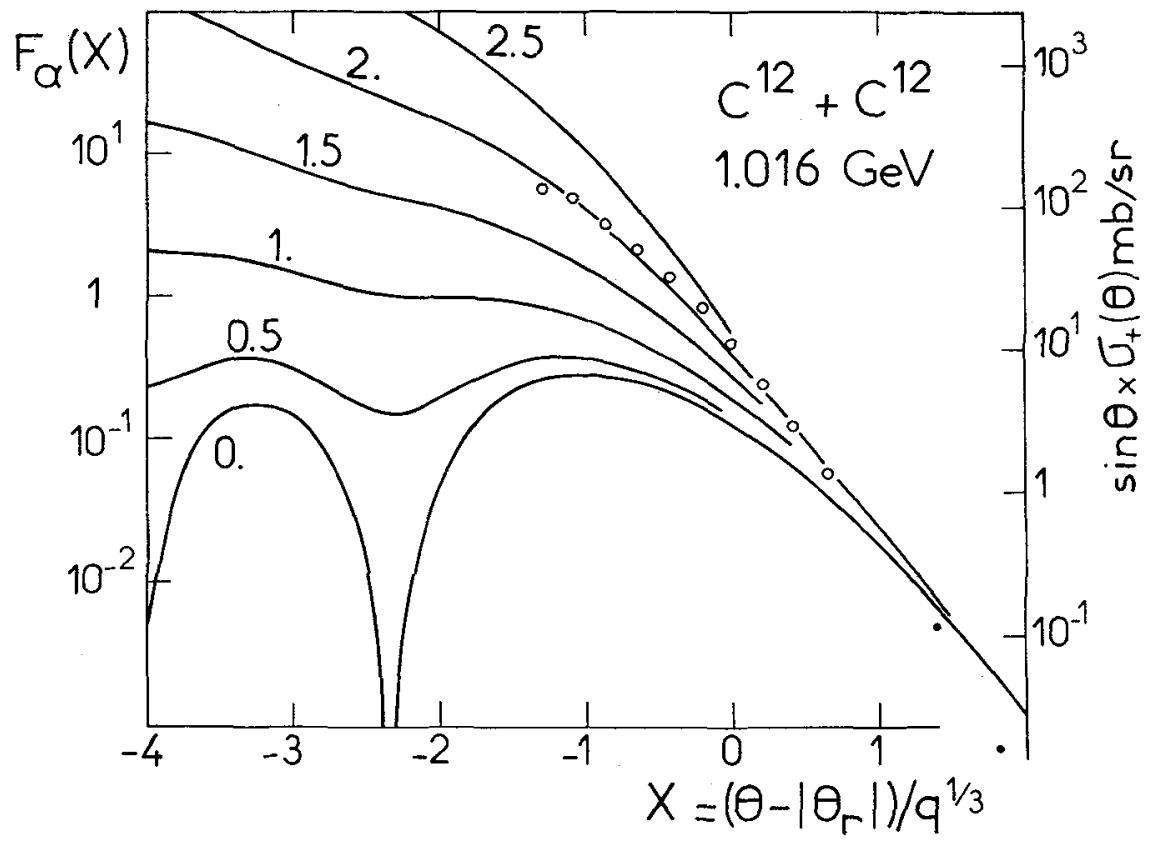

Fig. 3

This limit corresponds to a transparent nucleus. The resulting pattern is that of an Airy function which exhibits the familiar structure due to interferences of classical trajectories near a "rainbow". As absorption is introduced, $\alpha>0$, the oscillatory structure gradually disappears. However, it should be noted that for $\theta \tilde{>}\left|\theta_{r}\right|$ the effect of absorption is rather smal1. The asymptotic behaviour of $F_{\alpha}(x)$ illustrates these qualitative analysis. One has,

$$
\begin{aligned}
& \theta \ll\left|\theta_{r}\right|, \quad|x| \gg 1 \quad(x<0) \text { and } \alpha \approx 1 \\
& F_{\alpha}(x) \sim \frac{1}{4 \pi} \frac{\exp (2 \alpha \sqrt{|x|})}{\sqrt{|x|}}+\frac{1}{\pi \sqrt{|x|}} \sin ^{2}\left[\frac{2}{3}|x|^{3 n}+\pi / 4\right]
\end{aligned}
$$

This expression shows the strong dependency of the angular distribution pattern on the parameter $\alpha$, in the angular range $\theta<\left|\theta_{r}\right|$.

$$
\begin{aligned}
& \theta \gg\left|\theta_{r}\right|, \quad x \gg 1, \\
& F_{\alpha}(x) \approx \frac{1}{4 \pi} \frac{\exp \left(-\frac{4}{3} x^{3 / 2}\right)}{\sqrt{|x|}}
\end{aligned}
$$

This expression is independent of $\alpha$. This is an important result. It means that :

For $\theta S\left|\theta_{r}\right|$ the scattering cross section is weakly affected by absorption and therefore remains essentially determined by the nuclear rainbow effect. 
We now consider the particular case of $C^{12}+C^{12}$ scattering at $E=1.016 \mathrm{GeV}$. With the parameters of Buenerd et a1. $/ 2 /$, for $V(r)$, one has from (11) and (12) $\theta_{r}=10^{\circ}$, and $q=0.000547$. These define the reduced variable $x$. We have plotted in figure 3 the points attributed to the $\sigma_{+}(\theta)$ component (see figure 2) multiplied by $\sin \theta$. A reasonably good agreement is obtained with the curve $F_{\alpha}(x)$, for $\alpha=2$. This leads to $\Delta=6.11$ and, as $\ell_{r} \sim k R=44.2$, one obtains $\ell_{0}=59$ and $\exp \omega\left(\ell_{r}\right)=\left|S\left(\ell_{r}\right)\right|=$ $=0.089 \sim 0.1$. These values are very close to those obtained by a phase shift analys is of the data $/ 10 /$.

In the light of the arguments given above we can conclude with two main remarks : For $\theta \ll\left|\theta_{r}\right|$, the absorption strongly reduces the contribution of the inner branch $(\ell<\ell r)$ of the deflection function. As a result, the first "rainbow" maximum is masked.

For $\theta \check{>}\left|\theta_{r}\right|$, the exponential-like decrease observed in the scattering crosssection of $\mathrm{C}^{12}+\mathrm{C}^{12}$ at $1.016 \mathrm{GeV}$, is essentially a shadow effect due to a nuclear rainbow.

\section{REFERENCES}

/1/ BUENERD M. et a1., Phys. Lett. 102B (1981) 242.

12/ BUENERD M. et a1., Phys. Rev. C26 (1982) 1299.

/3/ GOLDBERG D.A. and SMITH S.M., Phys. Rev. 33 (1974) 715.

/4/ da SILVEIRA R., Phys. Lett. 45B (1973) 211.

/5/ FORD K. and WEELER J.A., Ann. of Phys. 7 (1959) 259

16/ da SILVEIRA R. and LECLERCQ-WILLAIN Ch. 7 . Phys. A314 (1983) 63.

/7/ GILS H.J. et al., Phys. Rev. C21 (1980) 1239.

18/ GLAUBER R.J., "Lectures in Theoretical Physics" Vol.I (1959) 315.

19/ BERRY M.V., Proc. Phys. Soc., 89 (1966) 479.

/10/ CONNOR J.N.L., J. Chem. Phys. 74 (1981) 1047.

/11/ FRAHN W.E. and VENTER R.H., Ann. of Phys. 241963) 243

112/ LANDAU L. and LIFCHITZ L., "Mécanique", Edítions de Moscou, 1960. da SILVEIRA R., Phys. Lett. 50B (1974) 237. 